\title{
Cholangiocarcinomas associated with long-term inflammation express the activation-induced cytidine deaminase and germinal center-associated nuclear protein involved in immunoglobulin V-region diversification
}

\author{
WARAPORN CHAN-ON ${ }^{1-3}$, KAZUHIKO KUWAHARA ${ }^{1}$, NAOYA KOBAYASHI ${ }^{4}$, KAZUTAKA OHTA ${ }^{1}$, \\ TATSUYA SHIMASAKI ${ }^{5}$, BANCHOB SRIPA ${ }^{6}$, CHANVIT LEELAYUWAT $^{2}$ and NOBUO SAKAGUCHI ${ }^{1,7}$ \\ ${ }^{1}$ Department of Immunology, Graduate School of Medical Sciences, Kumamoto University, Kumamoto, Japan; \\ ${ }^{2}$ Department of Clinical Immunology, The Centre for Research and Development of Medical Diagnostic Laboratories, \\ Faculty of Associated Medical Sciences, ${ }^{3}$ Biomedical Sciences Program, Khon Kaen University, Khon Kaen, Thailand; \\ ${ }^{4}$ Department of Surgery, Okayama University Graduate School of Medicine and Dentistry, Okayama; \\ ${ }^{5}$ Institute of Resource Development and Analysis (IRDA), Kumamoto University, Kumamoto, Japan; \\ ${ }^{6}$ Department of Pathology, Faculty of Medicine and Liver Fluke and Cholangiocarcinoma Research Center, \\ Khon Kaen University, Khon Kaen, Thailand; ${ }^{7}$ Core Research for Evolutional Science and \\ Technology Program (CREST), Japan Science and Technology Agency (JST), Japan
}

Received March 20, 2009; Accepted May 12, 2009

DOI: 10.3892/ijo_00000339

\begin{abstract}
Cholangiocarcinoma (CCA) represents a model of tumor development after long-term inflammation which causes DNA damage or impairs DNA repair mechanism. AID and GANP, both appearing in antigen-driven B cells, are involved in affinity maturation of the immunoglobulin $\mathrm{V}$ region with increased somatic mutation. A normal cholangiocyte line showed the induction of $A I D$ transcripts after stimulation with TNF- $\alpha$, whereas ganp transcripts appeared constitutively in this cell line. Next, we examined the expression of AID and GANP in clinical CCA specimens to
\end{abstract}

Correspondence to: Dr Nobuo Sakaguchi, Department of Immunology, Graduate School of Medical Sciences, Kumamoto University, 1-1-1 Honjo, Kumamoto 860-8556, Japan

E-mail: nobusaka@gpo.kumamoto-u.ac.jp

Dr Chanvit Leelayuwat, Department of Clinical Immunology, The Centre for Research and Development of Medical Diagnostic Laboratories, Faculty of Associated Medical Sciences, Khon Kaen University, Khon Kaen 40002, Thailand

E-mail: chanvit@kku.ac.th

Abbreviations: AID, activation-induced cytidine deaminase; CCA, cholangiocarcinoma; GC, germinal center; GANP, germinal center-associated nuclear protein; H. pylori, Helicobacter pylori; Ig, immunoglobulin; IHC, immunohistochemistry; mAb, monoclonal antibody; SHM, somatic hypermutation

Key words: cholangiocarcinoma, activation-induced cytidine deaminase, germinal center-associated nuclear protein, chronic inflammation, oncogenesis, $\gamma \mathrm{H} 2 \mathrm{AX}$, ionizing-irradiation, TNF- $\alpha$ obtain information whether their expression levels are associated with the malignant grade of CCA. AID expression was similarly detected in the clinical cases of both welldifferentiated and poorly-differentiated CCAs. On the contrary, GANP expression was detected in CCA cells at a higher level in the nucleus of poorly-differentiated CCAs with shorter survivals than in that of well-differentiated CCAs. The high and low cases of nuclear GANP expression showed no change in the frequency of the TP53 mutations, however, further investigation by in vitro experiment demonstrated that the high GANP expression caused the increased number of $\gamma \mathrm{H} 2 \mathrm{AX}$ foci after DNA damage by ionizing-irradiation. These results suggest that GANP is involved in regulation of DNA repair mechanism and the abnormal over-expression of GANP together with AID might be associated with rigorous DNA damage, potentially causing the malignant development of CCAs during long-term inflammation.

\section{Introduction}

Chronic infection with microorganisms including viruses, bacteria, and parasites is a serious risk factor of cancer (1). The high carcinogenic risk to humans infected with liver fluke or the bacterium Helicobacter $(H$.) pylori represents a peculiar clinical evidence prompting evaluation of the basis of the linkage between microbial infection and cancer development in humans. In Northeast Thailand, cholangiocarcinoma (CCA) occurs at a high incidence in patients infected with the liver fluke, Opisthorchis viverrini, implying that chronic inflammation of the hepatobiliary tract is associated with tumor development (2-5). The long-term inflammatory responses with the production of various proinflammatory cytokines presumably cause DNA damage or 
impair the DNA repair mechanism, thus contributing to carcinogenic transformation.

Activation-induced cytidine deaminase (AID) has been proposed to be a causative factor for TP53 mutations in patients infected with $H$. pylori, which indicates a close linkage of inflammation with gastric cancer (6). AID is induced specifically in germinal center (GC)-B cells and is an initiator of somatic hypermutation (SHM) of immunoglobulin (Ig) V-region genes and class switch recombination in vivo (7). AID catalyzes deamination of cytidine, generating the $\mathrm{C} \rightarrow \mathrm{U}$ alteration that is a key DNA change, and eventually causing $\mathrm{C} \rightarrow \mathrm{T}$ and $\mathrm{G} \rightarrow \mathrm{A}$ mutations in both DNA strands (8).

GC-associated nuclear protein (GANP) is also required for affinity maturation with increased SHM at the $I g V$-region after immunization $(9,10)$. GANP is a $210-\mathrm{kDa}$ nuclear protein expressed ubiquitously but up-regulated in GC-B cells (11-13). The middle portion of GANP is homologous to Saccharomyces cerevisiae SAC3, which is involved in mRNA export from the nucleus to the cytoplasm. SAC3-deficient cells demonstrate DNA hyper-recombination, as measured by using a tandem-repeat leu2 reporter gene (14); and similar activity has been demonstrated in GANP-deficient mouse cells (15). GANP is suggested to be involved in generation of SHM or modifying/repairing DNA injuries caused by cytidine deamination. Previously, we demonstrated that GANP expression is up-regulated in various hematological diseases and that the transgenic mice of ganp gene under the control of $I g$ promoter/enhancer developed lymphomas (16). However, it remained to investigate whether the increased GANP expression is associated with tumorigenesis and tumor development in clinical cancer cases. Here, we studied the expression of AID and GANP in CCAs, and examined whether their expressions change among the clinical grades of CCA development.

\section{Materials and methods}

CCA specimens. Surgically resected specimens were obtained from 65 patients with intrahepatic cholangiocarcinoma who were admitted to Srinagarind Hospital, Faculty of Medicine, Khon Kaen University, Thailand. Informed consents were obtained from all patients who participated in the project approved by Human Research Ethics Committee of Khon Kaen University. The data including age, gender, histological grading, and the involvement of lymph node, vascular and perineuron was reviewed retrospectively based upon medical records and pathologic records from the Liver Fluke and Cholangiocarcinoma Research Center, Khon Kaen University. Survival of patients was recorded from the date of surgery to the date of death or to March 13, 2007. Five patients were excluded from the survival analysis; two were lost to follow-up and three patients died within a month after surgery (perioperative death).

Cell lines. Human cell lines were maintained in suitable culture medium with $10 \%$ heat-inactivated fetal calf serum (Dainippon Pharmaceutical, Osaka, Japan), 2 mM L-glutamine (Cambrex, Charles City, IA), and $5 \times 10^{-5} \mathrm{M}$ 2-mercaptoethanol in a humidified incubator with $5 \% \mathrm{CO}_{2}$ at $37^{\circ} \mathrm{C}$ : CCA lines [OCA17, M055, and KKU-100; established from patients with liver fluke-related CCA in Department of Pathology, Faculty of Medicine, Khon Kaen University (17)] in Ham-F12 (Invitrogen, Carlsbad, CA), the immortalized cholangiocyte line MMNK-1 (18) in DMEM (Invitrogen), and lymphoid cell lines (Ramos and Jurkat) in RPMI-1640 (Invitrogen). NIH-3T3/pEF and NIH-3T3/pEF-ganp transfectants were established by using PolyFect (Qiagen, Valencia, CA) and cultured in DMEM containing hygromycin B (Invitrogen).

Reverse transcriptase $(R T)-P C R$. The cDNA was prepared by SuperScript III reverse transcriptase (Invitrogen) with total RNAs from cells cultured in vitro or from frozen CCA tissues. AID transcripts were amplified by oligonucleotide primers based on the information of a previous study (19). RT-PCR for AID was carried out using Z-Taq DNA polymerase (Takara Bio, Otsu, Japan), the amplification conditions were 35 cycles of $98^{\circ} \mathrm{C}$ for $10 \mathrm{sec}, 58^{\circ} \mathrm{C}$ for $10 \mathrm{sec}$, and $72^{\circ} \mathrm{C}$ for $40 \mathrm{sec}$. Ganp was amplified using AmpliTaq Gold DNA polymerase (Applied Biosystems, Foster City, CA) at $95^{\circ} \mathrm{C}$ for $9 \mathrm{~min}$ followed by 35 cycles of $94^{\circ} \mathrm{C}$ for $20 \mathrm{sec}, 57^{\circ} \mathrm{C}$ for $20 \mathrm{sec}, 72^{\circ} \mathrm{C}$ for $30 \mathrm{sec}$ and final extension at $72^{\circ} \mathrm{C}$ for $5 \mathrm{~min}$ (12). $\beta$-actin transcripts were amplified as internal controls with 23 cycles of $98^{\circ} \mathrm{C}$ for $5 \mathrm{sec}, 55^{\circ} \mathrm{C}$ for $10 \mathrm{sec}$, and $72^{\circ} \mathrm{C}$ for $10 \mathrm{sec}$ (12). AID and ganp transcripts were verified by blot transfer and hybridization with human $A I D$, ganp, and $\beta$-actin cDNA probes.

Real-time RT-PCR. Real-time RT-PCR was conducted by using a LightCycler 2.0 Instrument (Roche Applied Science, Indianapolis, IN) and a pair of Hybridization Probes labeled either at the 5'-end with a LightCycler Red fluorophore or at the 3'-end with fluorescein and LightCycler DNA Master HybProbe (Roche Diagnostics, Indianapolis, IN). Specific oligonucleotide primers were synthesized according to published information on the AID and ganp genes as follows: AID sense, 5'-TGGTGGGACGACAAACTG-3'; AID antisense, 5'-GTCCCAGTCCGAGATGTAG-3'; ganp sense, 5'-CGTGGAGCTGATGGAACG-3'; ganp anti-sense, 5'-GCA GAAGCACTGAAGCTCCT-3'. For AID, the donor probe 5'-CCGTTCTTATTGCGAAGATAACCAAAGTCCAG-3' was labeled with fluorescein at its 3 '-end, whereas the acceptor probe 5'-GAAAAGGATGTAGCACTGTCACGCC TCTTCA-3' was labeled with LC Red 640 at its 5'-end. For ganp, the donor probe 5'-AGTGGGCACAGACATCCTCAC AGCAACG-3' and the acceptor probe 5'-GCCACACGGAC CCTCTGGTCTGTCTCTA-3' were prepared. To ensure the fidelity of mRNA extraction and reverse transcription, all samples were subjected to PCR amplification with oligonucleotide primers and probes specific for the constitutively expressed gene glyceraldehyde-3-phosphate dehydrogenase (gapdh) and normalized. The gapdh primers used were as follows: forward, 5'-CAGCCTCAAGATCATCAGC-3'; reverse, 5'-GGCCATCCACAGTCTTCT-3'. The sequences of the gapdh probes used for real-time LightCycler PCR were 5'-GGTCATCCATGACAACTTTGGTATCGTGGAA-3' and 5'-GACTCATGACCACAGTCCATGCCATCACTG-3'. All primers and probes were purchased from Nihon Gene Research Laboratories Inc. (Sendai, Japan). The cycle conditions for real-time RT-PCR were: $A I D, 95^{\circ} \mathrm{C}$ for $1 \mathrm{~min}$, followed by 50 cycles of $95^{\circ} \mathrm{C}$ for $0 \mathrm{sec}, 56^{\circ} \mathrm{C}$ for $5 \mathrm{sec}$, and 
$72^{\circ} \mathrm{C}$ for $5 \mathrm{sec}$; ganp, $95^{\circ} \mathrm{C}$ for $1 \mathrm{~min}$, then 50 cycles of $95^{\circ} \mathrm{C}$ for $0 \mathrm{sec}, 55^{\circ} \mathrm{C}$ for $5 \mathrm{sec}$, and $72^{\circ} \mathrm{C}$ for $5 \mathrm{sec}$; gapdh, $95^{\circ} \mathrm{C}$ for $1 \mathrm{~min}$, followed by 45 cycles of $95^{\circ} \mathrm{C}$ for $0 \mathrm{sec}, 55^{\circ} \mathrm{C}$ for $5 \mathrm{sec}$, and $72^{\circ} \mathrm{C}$ for $7 \mathrm{sec}$.

Immunohistochemistry (IHC). Paraffin sections (4- $\mu \mathrm{m})$ were immunostained with rat anti-GANP monoclonal $\mathrm{Ab}(\mathrm{mAb})$ (11) or rat anti-AID mAb (EK2 5G9; Cell Signaling Technology, Danvers, MA) in combination with biotinylated anti-rat $\mathrm{Ig} \mathrm{Ab}$ with Vectastain ABC complex (Vector Laboratories, Burlingame, CA) and 3,3'-diaminobenzidine tetrahydrochloride (Sigma-Aldrich, St. Louis, MO). Human tonsil tissue was used as a positive control. The expression level was evaluated blindly by 2 investigators as IHC score by multiplication of positivity $(0-100 \%)$ and the staining intensity $(0$, no; 1 , weak; 2 , moderate; and 3 , strong).

Immunostaining by anti-GANP $m A b$. Three CCA lines were cultured in Lab-Tek chamber slides (Nalge Nunc International, Rochester, NY) at $37^{\circ} \mathrm{C}$ overnight. After fixation with $3.7 \%$ paraformaldehyde/PBS and permeabilization with $0.2 \%$ Triton X-100, cells were incubated with anti-GANP mAb as a primary Ab. Histofine Simple Stain Mouse MAX PO (Nichirei Biosciences Inc., Tokyo, Japan) was used as a secondary Ab. After the development by 3,3'-diaminobenzidine tetrahydrochloride, cells were lightly counterstained by hematoxylin.

Western blot analysis. MMNK-1 cells with ganp RNAi-treatment or NIH-3T3/pEF-ganp transfectants were harvested by trypsinization, washed twice in cold PBS, and lyzed with TNE buffer (10 mM Tris-HCl, pH 7.4, $150 \mathrm{mM} \mathrm{NaCl}, 1 \%$ Nonidet P-40, and $1 \mathrm{mM}$ EDTA, supplemented with protease inhibitor cocktails and $1 \mathrm{mM}$ PMSF). After clearing by centrifugation at $15,000 \mathrm{rpm}$ for $15 \mathrm{~min}$ at $4^{\circ} \mathrm{C}$, lysates in SDS sample buffer were heated for $5 \mathrm{~min}$ at $98^{\circ} \mathrm{C}$, separated by SDS-PAGE and transferred to nitrocellulose membranes. Blots were washed in PBS containing 0.1\% Tween-20 and then blocked with 5\% non-fat dry milk in PBS/0.1\% Tween-20. The anti-AID and anti-Flag (M2; Stratagene, La Jolla, CA) mAbs were used in combination with horseradish peroxidaseconjugated secondary $\mathrm{Ab}$ and an enhanced chemiluminescence kit (GE Healthcare, Piscataway, NJ). B-actin was used as a loading control.

Subcloning and sequencing of human TP53 gene. Human TP53 was amplified from the genomic DNA in non-transcribed (nucleotide positions 1-842) and transcribed (exons 5-8) regions by using Pfu-Turbo DNA polymerase (Stratagene), subcloned into pENTR/D-TOPO (Invitrogen), and sequenced using ABI PRISM 310 Genetic Analyzer (Applied Biosystems). The TP53-specific primers used were as follows: untranscribed, forward, 5'-TTCCCATCAAGCCCTAGG GCT-3'; reverse, 5'-TTTTGAGAAGCTCAAAACTTTTA GC-3'. Transcribed, forward, 5'-TACTCCCCTGCCCTCAA CAAGATGTT-3'; reverse, 5'-CTCGCTTAGTGCTCCCT GGG-3'.

Ganp RNAi-treatment. MMNK-1 cells were transfected with ganp RNAi (10 $\mathrm{nM})$ in the presence of Lipofectamine
RNAiMAX (Invitrogen) according to the manufacturer's protocols. The following Stealth RNAi duplexes were synthesized by Invitrogen: for ganp RNAi-1, sense, 5'-CCAGCGU CUUCUGGAGUAAGUCAUU-3' and anti-sense, 5'-AAUG ACUUACUCCAGAAGACGCUGG-3' were used and for scrambled control RNAi, sense, 5'-CCCACCUCAAGUGUU GGACCAACUU-3' and anti-sense, 5'-AAGUUGGUCCAA CACUUGAGGUGGG-3' were used.

Cell cycle analysis. Cell cycle was analyzed with PI by using a FACSCalibur (Becton-Dickinson, San Jose, CA) as described previously (13).

Immunofluorescence of $\gamma H 2 A X$. After $3 \mathrm{~h}$ of $1-\mathrm{Gy}$ irradiation, $\gamma \mathrm{H} 2 \mathrm{AX}$ foci were detected by using anti-phospho-histone H2AX (Ser139) mAb (Millipore, Temecula, CA) and Alexa Fluor 488-conjugated anti-mouse $\mathrm{IgG} \mathrm{Ab}$ (Invitrogen) with 4,6-diamidino-2-phenylindole staining. The number of foci in 20 cells was counted under a fluorescent microscope (BX51; Olympus, Tokyo, Japan).

Statistical analysis. AID and GANP were compared between the clinical groups classified with various parameters by statistical analysis using SPSS15.0 software for MannWhitney's U test (Chicago, IL). Patient survival was calculated from the time of resection to either death or the last follow-up. The survival curves were assessed by the Kaplan-Meier analysis using a log-rank test. $\mathrm{P}<0.05$ was considered statistically significant.

\section{Results}

Expression of AID and GANP at various CCA stages. CCA lines of well-differentiated (OCA17), moderately-differentiated (M055), and poorly-differentiated (KKU-100) tumors (17) were examined for their expression of AID and ganp transcripts. AID was not detected in OCA17; however, it was transcribed highly in M055 and KKU-100 cells (Fig. 1A). The ganp transcript level also increased with the progression of the malignancy grade (Fig. 1A). The AID transcript level was 10- and 15-fold higher in M055 and KKU-100 cells, respectively, than in well-differentiated OCA17 cells, as assessed by real-time RT-PCR (Fig. 1B). The ganp transcript level was increased in M055 (70-fold) and KKU-100 (22-fold) cells, but was not detectable in OCA17 cells (Fig. 1C).

Next, we examined the expression of AID and GANP proteins by immunostaining (Fig. 1D). AID expression was strong in M055 and KKU-100 (b and c) and moderate in OCA17 (a) cells, appearing in the cytoplasm but occasionally also in the nucleus (arrowheads) in comparison with that in Jurkat (d, negative control), Ramos (e, positive control), and tonsillar GC-B (f, positive control) cells. GANP was seen mostly in the nuclei of CCA lines (Fig. 1E). The expression levels of GANP in M055 and KKU-100 were much stronger than that observed in OCA17. Thus AID and GANP appeared in the development of CCA, and their expression levels were high in less-differentiated CCAs.

AID transcription in normal cholangiocytes by TNF- $\alpha$ stimulation. We analyzed the transcripts of AID and ganp 


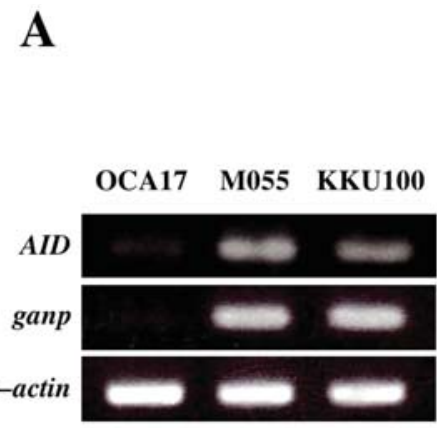

B

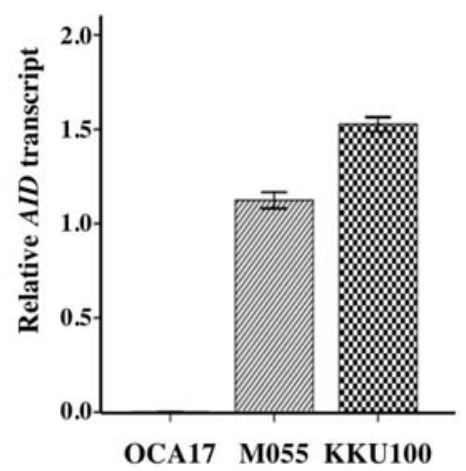

C

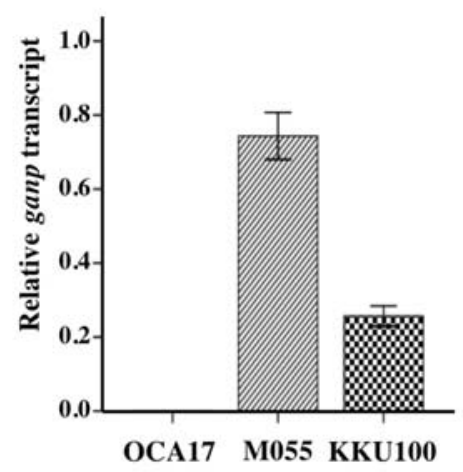

D
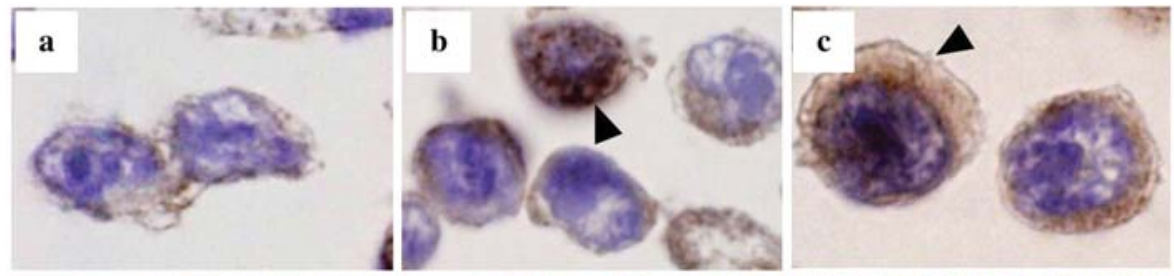

1000x

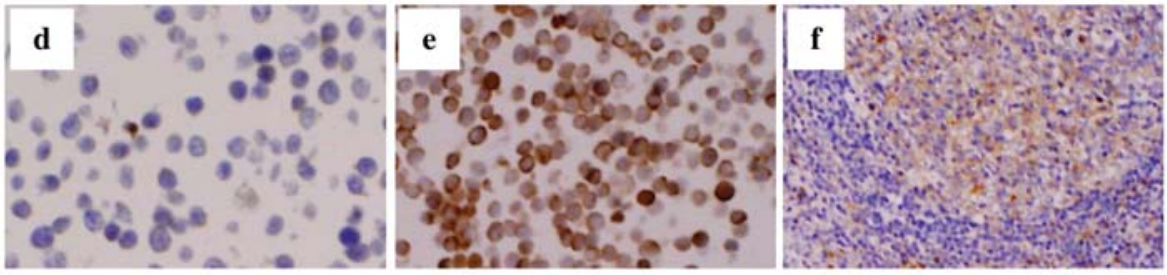

200x

E
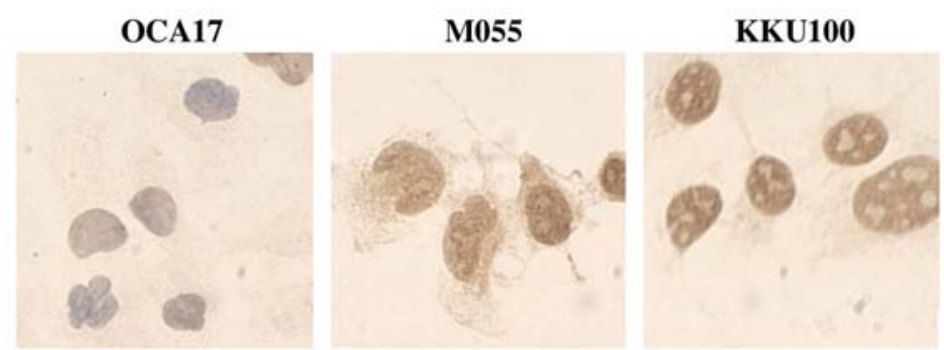

1000x

Figure 1. AID and GANP expression levels in CCA lines. (A) RT-PCR results for AID and ganp transcripts are shown with $\beta$-actin control. (B and C) AID (B) and ganp (C) transcripts were examined by real-time RT-PCR, and their levels are presented as the mean \pm SE from 3 independent experiments. (D) Immunostaining of AID in CCA cell lines. AID was detected throughout the cytoplasm, and occasionally in the nucleus, of M055 (b) and KKU-100 (c) at a higher level than in OCA17 cells (a). Arrowheads indicate cells expressing AID in both nucleus and cytoplasm in comparison with the expression in Jurkat (d) and Ramos (e) or human tonsillar GCs (f). (E) Immunostaining of GANP in CCA cell lines. GANP expression was predominantly detected in nuclei of cells.

after treatment of a normal cholangiocyte line, MMNK-1, with TNF- $\alpha$. The AID transcript level was low in MMNK-1 cells but became elevated after stimulation with TNF- $\alpha$ (Fig. 2A), whereas ganp transcription was almost constitutive in the cells as shown in the relative fold expression (Fig. 2A, lower panel). AID expression at the protein level was markedly increased in MMNK-1 after TNF- $\alpha$ stimulation by Western blot analysis (Fig. 2B, upper panel) and immunocytostaining (Fig. 2B, lower panel), suggesting that an aberrant increase of AID expression occurred after TNF- $\alpha$ stimulation in cholangiocytes that constitutively express GANP.

Expression of AID and GANP in liver fluke-related CCA samples. Next we studied whether AID and GANP were expressed in clinical cases of liver fluke-associated CCA by
RT-PCR. AID and ganp transcripts were detected in all of CCA samples from 21 cases (Fig. 3A). CCA specimens (65 cases) showed higher AID expression similarly in tumors than in the surrounding stromal cells as shown in the representative cases (Fig. 3B, a and c). GANP was expressed in CCAs but with the different expression profiles of two types, i.e., as a cytoplasm-dominant (GANP ${ }^{\text {cyto }}$ ) type (Fig. 3B, b) and as a nucleus-dominant (GANPucl) one (Fig. 3B, d). These results demonstrated that AID was aberrantly expressed in nonlymphoid tumor CCAs as reported previously $(6,20)$ and additionally GANP was co-expressed, but with different expression levels in the CCA cells.

Correlation of AID and GANP with clinicopathological findings. The clinical significance of AID and GANP expres- 
A

\begin{tabular}{|c|c|c|c|c|c|c|}
\hline & \multicolumn{6}{|c|}{ Time (h) } \\
\hline & 0 & 4 & 6 & 8 & 10 & 24 \\
\hline$A I D$ & 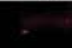 & $=0$ & -2 & 20 & tatay & $m=$ \\
\hline ganp & $\infty$ & $=$ & $=$ & $=$ & 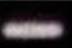 & $=$ \\
\hline -actin & $=$ & 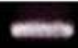 & $\Rightarrow$ & = & $=$ & 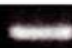 \\
\hline
\end{tabular}
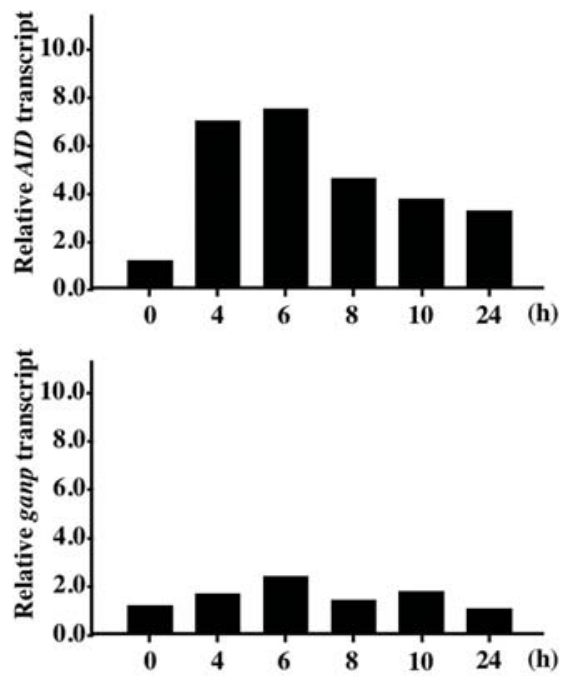

B

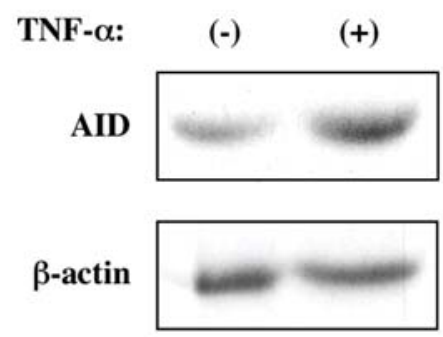
Non-stimulated
MMNK-1
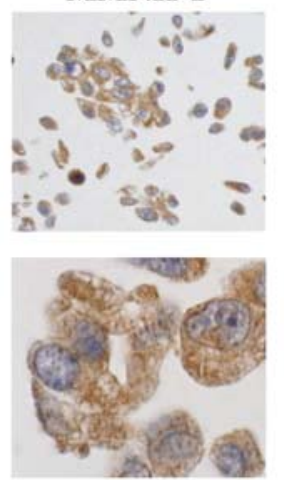

TNF- $\alpha$-stimulated MMNK-1

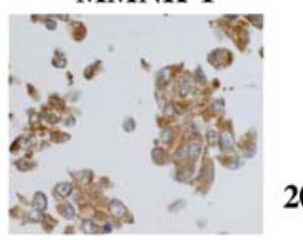

$200 x$

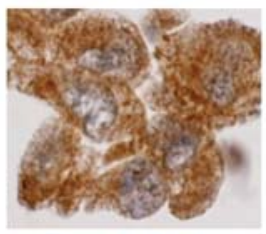

$1000 x$

Figure 2. Induction of AID in normal cholangiocytes by TNF- $\alpha$. (A) Transient induction of AID, but not ganp, in TNF- $\alpha$-stimulated MMNK-1 cells. MMNK-1 cells were stimulated with TNF- $\alpha(100 \mathrm{ng} / \mathrm{ml})$ for indicated times, and semi-quantitative RT-PCR was performed using specific primer for AID, ganp, and $\beta$-actin (upper panel). Real-time RT-PCR of AID and ganp transcripts was performed using same samples as (A) (lower panels). (B) Increased expression of AID in TNF- $\alpha$-stimulated MMNK-1 cells. MMNK-1 cells were stimulated with or without TNF- $\alpha(100 \mathrm{ng} / \mathrm{ml})$ for $8 \mathrm{~h}$, and immunoblot (upper panel) and immunostaining (lower panel) of AID were performed.

\section{A}

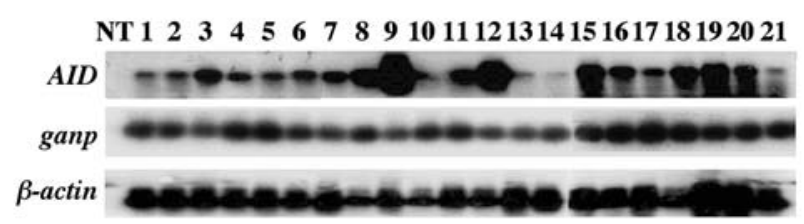

B

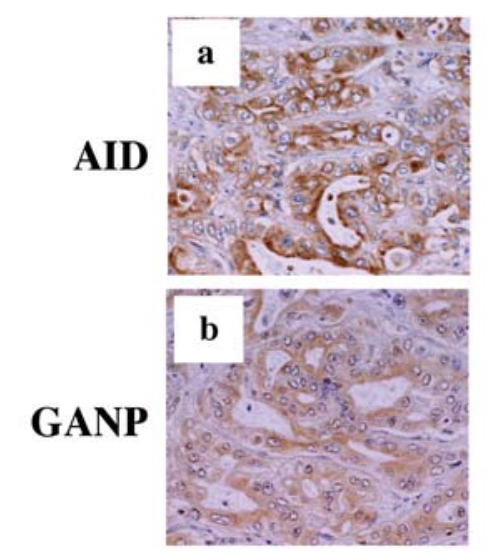

Case 1
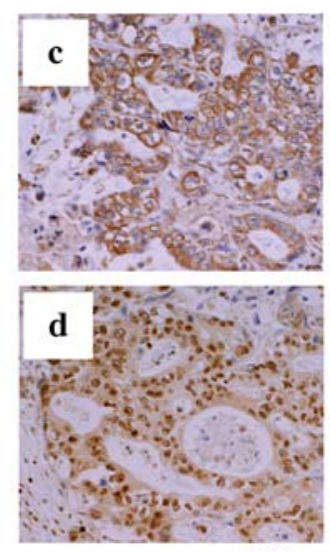

200x

Figure 3. Expression of AID and GANP in liver fluke-related CCA tissues. (A) RT-PCR of AID and ganp transcripts in patients' samples. NT, non-template. (B) Immunostaining of AID and GANP in CCA samples. AID was exclusively detected in cytoplasms (a and c), whereas GANP was found in cells as a cytoplasm-dominant (b) or a nucleus-dominant type (d).

sions was evaluated by IHC in comparison with clinicopathological parameters (Table I). Levels of AID and GANP ${ }^{\text {nucl }}$ in CCA specimens were $133 \pm 44$ and $44 \pm 47$ (mean \pm SD), respectively. AID was not associated with any parameters significantly. However, the expression of GANPucl showed a significant correlation with the histological types of CCA $(\mathrm{p}=0.037)$. GANPnucl was up-regulated in less-differentiated CCAs $(63 \pm 59$; mean $\pm \mathrm{SD})$, the level was significantly higher 
Table I. Correlation between the staining of GANP and AID and the clinicopathological factors.

\begin{tabular}{|c|c|c|c|c|c|}
\hline \multirow[b]{2}{*}{ Parameters } & \multirow[b]{2}{*}{$N(65)$} & \multicolumn{2}{|c|}{ Nuclear GANP } & \multicolumn{2}{|c|}{ Cytoplasmic AID } \\
\hline & & IHC score $($ mean $\pm \mathrm{SD})$ & p-value & IHC score $($ mean $\pm \mathrm{SD})$ & p-value \\
\hline Age (year) & & & NS & & NS \\
\hline$>50$ & 48 & $38 \pm 42$ & & $138 \pm 47$ & \\
\hline$\leq 50$ & 17 & $61 \pm 57$ & & $120 \pm 33$ & \\
\hline Gender & & & NS & & NS \\
\hline Male & 44 & $37 \pm 44$ & & $131 \pm 42$ & \\
\hline Female & 21 & $58 \pm 50$ & & $137 \pm 50$ & \\
\hline Histological type & & & $0.037^{\mathrm{a}}$ & & NS \\
\hline Well-differentiated & 45 & $35 \pm 39$ & & $136 \pm 43$ & \\
\hline Less-differentiated & 20 & $63 \pm 59$ & & $127 \pm 47$ & \\
\hline Lymph node metastasis & & & NS & & NS \\
\hline Positive & 23 & $34 \pm 48$ & & $135 \pm 34$ & \\
\hline Negative & 42 & $49 \pm 46$ & & $132 \pm 49$ & \\
\hline Neural invasion & & & NS & & NS \\
\hline Positive & 3 & $27 \pm 22$ & & $140 \pm 99$ & \\
\hline Negative & 62 & $45 \pm 48$ & & $133 \pm 42$ & \\
\hline Vascular invasion & & & NS & & NS \\
\hline Positive & 14 & $68 \pm 66$ & & $144 \pm 70$ & \\
\hline Negative & 51 & $37 \pm 39$ & & $130 \pm 35$ & \\
\hline
\end{tabular}

Well-differentiated, papillary and well-differentiated adenocarcinoma; less-differentiated, moderately- and poorly-differentiated

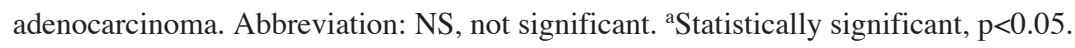

A

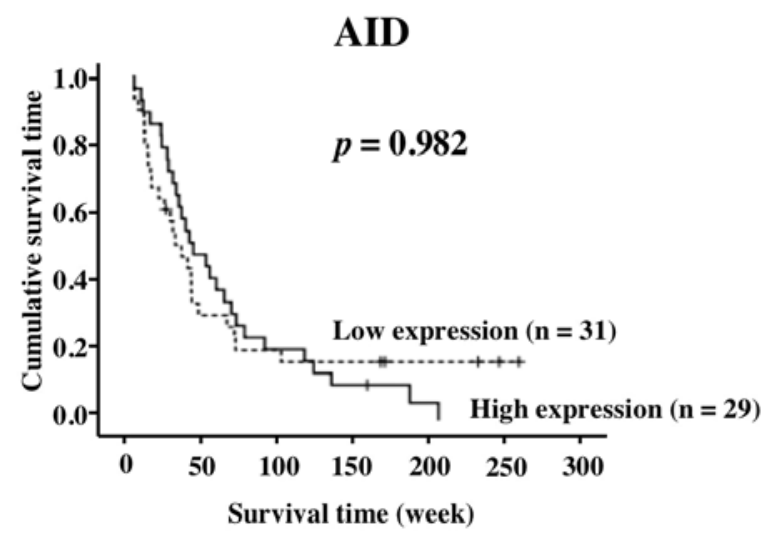

B

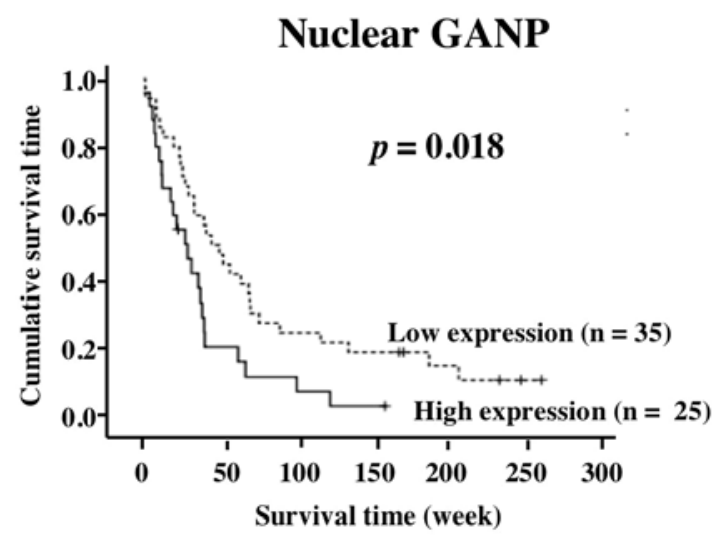

Figure 4. Survival of patients with GANP and AID expression. (A) The patient survival was compared with the expression levels of AID. (B) The survival rate of patients with high expression of GANP ${ }^{\text {nucl }}$ was compared.

than that in well-differentiated type $(35 \pm 39$; mean $\pm \mathrm{SD})$. The increased expression of nuclear GANP might be associated with genetic and cellular changes in CCA.
Expression of AID and GANP, and cumulative survival. CCA patients with high AID expression showed shorter survival times, but the difference was not statistically significant 
A
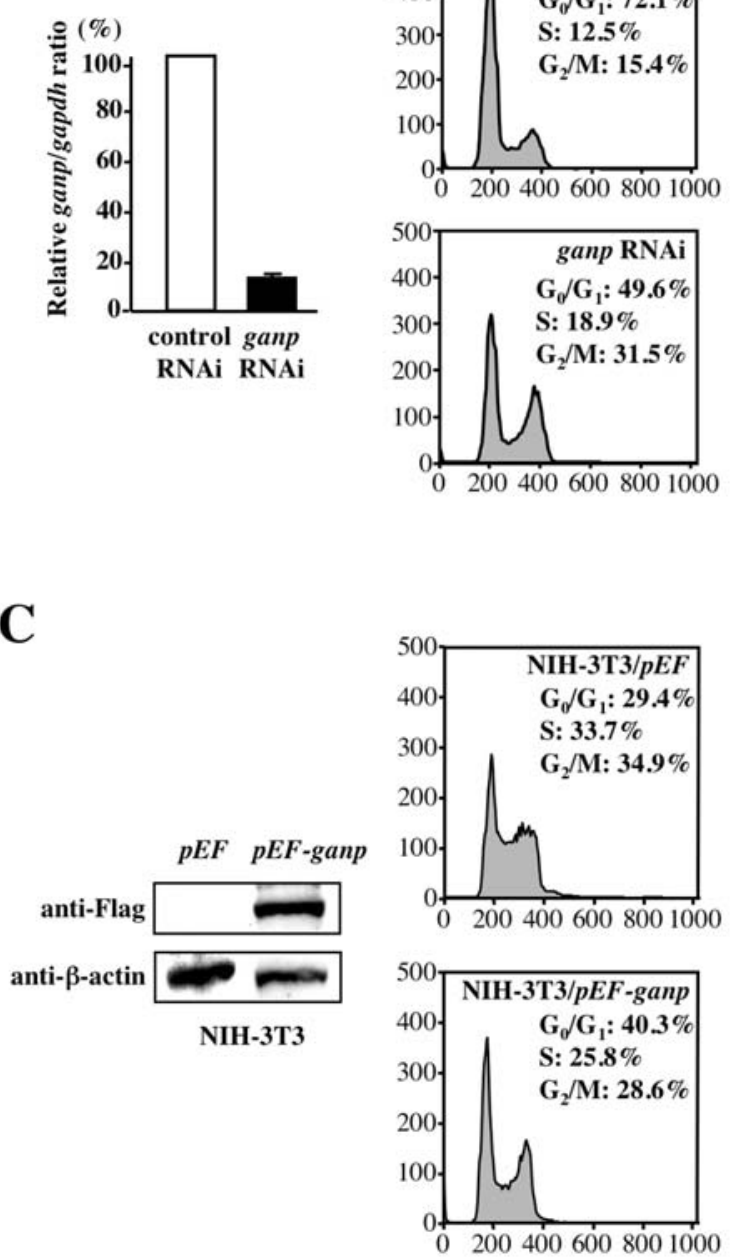

C
B

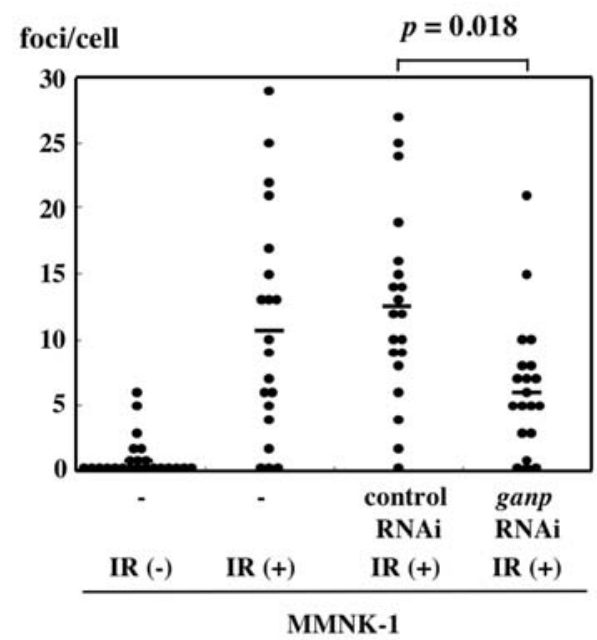

D

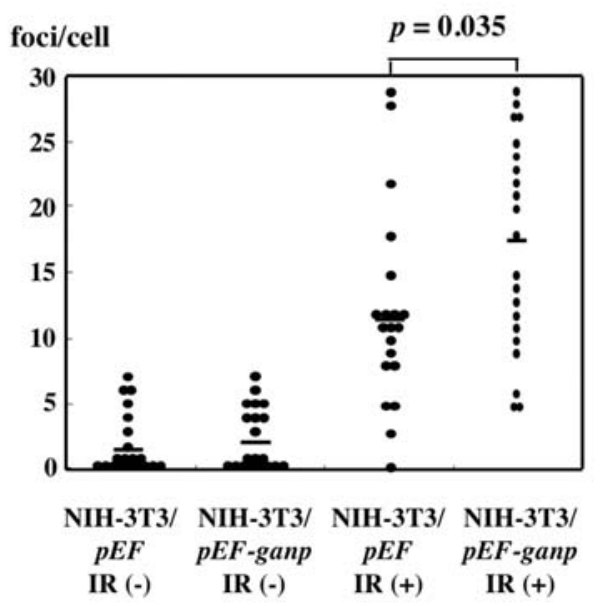

Figure 5. Regulation of DNA repair by GANP. (A) MMNK-1 cells with ganp RNAi-treatment. The knockdown efficiency of ganp transcripts was confirmed by real-time RT-PCR (left panel). The cell-cycle progressions in control and ganp RNAi-treated MMNK-1 were analyzed by PI staining (right panels). (B) The generation of $\gamma \mathrm{H} 2 \mathrm{AX}$ foci was examined in the ganp RNAi-treated cells (loss-of-function) after ionizing-irradiation (1 Gy). The average numbers of foci/cell are shown with the bars. (C) Establishment of NIH-3T3 cells over-expressing ganp cDNA. The introduced GANP was clearly detected in the cell line by anti-Flag immunoblot (left panel). The cell-cycle progressions in NIH-3T3/pEF and NIH-3T3/pEF-ganp were analyzed by PI staining (right panels). (D) The effects of ganp cDNA transfection (gain-of-function) were examined in NIH-3T3 cells (B). The average number of $\gamma \mathrm{H} 2 \mathrm{AX}$ foci indicated with the bar was compared.

(Fig. 4A). Aberrant expression of AID was observed in CCA specimens, but the difference in AID level may not be directly associated with the progress and clinical course ( $\mathrm{p}=0.982$, log-rank test). However, the patients with high GANP ${ }^{\text {nucl }}$ expression showed a significantly shorter survival time than those with low GANP ${ }^{\text {nucl }}$ expression ( $\mathrm{p}=0.018$, log-rank test; Fig. 4B). Thus, high expression of GANPnucl may be a useful prognostic indicator for malignant grades of CCAs.

Mutations of TP53 in CCA. CCA showed nuclear accumulation of TP53 in $25-75 \%$ of cases, and also displayed mutation hot-spots in exons 5-8 (21). In liver fluke-associated CCAs (55 cases), the loss of heterozygosity and the microsatellite instability of TP53 gene were reported to occur in 32\% (22). The predominant mutation pattern was $\mathrm{G}: \mathrm{C} \rightarrow \mathrm{A}: \mathrm{T}$ (at $\mathrm{CpG}$ ) in $29 \%$ of those cases. We examined whether expression of AID and GANP augmented the generation of TP53 mutations. The mutation frequency of transcribed and non- transcribed regions of the TP53 gene was compared between the $\mathrm{AID}^{+} \mathrm{GANP}^{\mathrm{L} o}$ and $\mathrm{AID}^{+}$GANPucl ${ }^{\text {nases }}$ (Table II). This genetic analysis could not detect any obvious difference in the frequency of TP53 mutations between the GANPLo and GANP ${ }^{\text {nucl }}$ groups, which might suggest that the effect of GANP over-expression is not limited to TP53 gene.

Effect of GANP on DNA damage caused by ionizing-irradiation. To explore the effect of GANP over-expression on cell proliferation in response to the genetic change that might have been caused by AID, we examined the cell cycle progression and the DNA damage caused by the change of GANP expression using the in vitro experimental systems. The ganp RNAi-treated cells showed a marked increase in the proportion of $\mathrm{G} 2 / \mathrm{M}$ phase cells (31.5\% of ganp RNAi cells vs. $15.4 \%$ of control RNAi cells), indicating that ganp is necessary for normal cell cycle progression of MMNK-1 cells (Fig. 5A, right panel); and these cells generated fewer $\gamma \mathrm{H} 2 \mathrm{AX}$ foci (6.1 foci/cell) compared with control cells 
Table II. Mutations of TP53 gene in CCA tumors.

\begin{tabular}{lccccc}
\hline & \multicolumn{2}{c}{ Untranscribed region } & & \multicolumn{2}{c}{ Transcribed (exon 5-8) region } \\
\cline { 2 - 2 } $\begin{array}{l}\text { CCA case } \\
\text { with }\end{array}$ & $\begin{array}{l}\text { No. of mutation/ } \\
\text { no. of sequence }\end{array}$ & $\begin{array}{c}\text { Mutation frequency } \\
\left(/ 10^{4} \mathrm{bp}\right)\end{array}$ & & $\begin{array}{c}\text { No. of mutation/ } \\
\text { no. of sequence }\end{array}$ & $\begin{array}{c}\text { Mutation frequency } \\
\left(/ 10^{4} \mathrm{bp}\right)\end{array}$ \\
\hline AID $^{+}$GANP & $0 / 6$ & 0.2 & $3 / 6$ & 0.3 \\
AID $^{+}$GANP & $0 / 5$ & 0.2 & $5 / 5$ & 0.5 \\
\hline
\end{tabular}

(12.6 foci/cell) upon the DNA damage caused by ionizingirradiation (Fig. 5B). These results suggest that GANP plays a role to accelerate the DNA damage response after the DNA injuries induced by ionizing-irradiation or AID cytidine deaminase activity. Thus, the effect of GANP over-expression was further investigated in NIH-3T3 cells in the absence of AID expression. The ganp over-expression in NIH-3T3 cells did not cause an increase of G2/M phase cells compared with mock-transfected cells (Fig. 5C, right panel), however, it caused the increase in the number of $\gamma \mathrm{H} 2 \mathrm{AX}$ foci $[17.5 \%$ of NIH-3T3/pEF-ganp $\left(\mathrm{IR}^{+}\right)$vs. $11.7 \%$ of NIH-3T3/pEF $\left.\left(\mathrm{IR}^{+}\right)\right]$ (Fig. 5D). This increased number of $\gamma \mathrm{H} 2 \mathrm{AX}$ foci by the overexpression of ganp cDNA was not detected without ionizingirradiation [2.2\% of NIH-3T3/pEF-ganp (IR') vs. $1.7 \%$ of NIH-3T3/pEF (IR-)]. Collectively, GANP is necessary for cell cycle progression, presumably through regulation of the DNA repair pathway after DNA double-strand breaks.

\section{Discussion}

AID and GANP, both involved in Ig diversification $(7,9)$, are expressed in liver fluke-related CCA. AID has been characterized as an activation-induced enzyme expressed specifically in B cells (7). AID was expressed and its expression was enhanced in MMNK-1 cells after stimulation with TNF- $\alpha$, suggesting that aberrant AID expression occurs under inflammatory conditions (Fig. 2), which is compatible to a previous report (20). On the contrary, GANP expression was already detected in MMNK-1 cells in the absence of TNF- $\alpha$, but we could not detect the enhancement of GANP expression in MMNK-1 cells in vitro (Fig. 2).

AID is expressed after $H$. pylori infection in gastric adenomatous cells (6), implying that AID might generate mutations of oncogenes and tumor suppressor genes. The fact that AID was expressed in cancerous cells suggested that the aberrant expression of AID unfavorably causes the cytidine deamination leading to accumulation of mutations in various critical genes of cell survival and cell cycle progression. Likewise, GANP was expressed in CCA at a particularly high level in less-differentiated CCA, which further suggested that both AID and GANP might play a part in tumor development.

AID is induced by $H$. pylori and is involved in the generation of TP53 mutations (6). Nevertheless, the mutation analysis of TP53 gene did not show any marked differences in the frequencies or mutation profiles between the GANPLo and GANP ${ }^{\text {nucl }}$ CCA cases, both expressing AID (Table II). GANP over-expression increased the generation of $\gamma \mathrm{H} 2 \mathrm{AX}$ foci (Fig. 5D), which might be associated with rigorous alteration of the genome rather than the increased accumulation of TP53 mutation. These observations were supported by the molecular properties of a GANP-homologue SAC3 in Saccharomyces cerevisiae (23). SAC3 is involved in mRNA export from the nucleus, and the lack of SAC3 causes DNA hyper-recombination by a homologous recombination mechanism at DNA injuries generated in the artificial reporter gene (14). The mechanism of DNA hyper-recombination triggered by the lack of SAC3 has not yet been elucidated, but it is thought that the lack of SAC3, causing impaired mRNA export from the nucleus as the hrRNP complex, generated DNA injuries (24). The decreased expression of mammalian GANP also caused homology-mediated DNA hyper-recombination generated by the ectopic expression of AID in NIH-3T3 cells (15), suggesting that GANP coordinately functions in the regulation of DNA injuries caused by AID.

Here, our results support a model of carcinogenesis associated with chronic infection of pathogenic microorganisms as a high-risk factor for cancer development. CCA might represent clinical cases of inflammation-associated oncogenesis. GANP, which regulates the process of the DNAmodifying/repairing mechanism after DNA injury, is closely associated with AID in tumorigenesis and tumor development of cholangiocytes during the long-term inflammation caused by a liver fluke infection.

\section{Acknowledgements}

We thank Ms. Y. Fukushima and Ms. Y. Kumamoto for excellent assistance. This work was supported by a Grant-inaid from the Ministry of Education, Culture, Sports, Science, and Technology (K.K. and N.S.), JSPS Asia-Africa Science Platform Program (N.S.), CREST of Japan Science and Technology Agency (N.S.) in Japan, and the Royal Golden Jubilee PhD Program (PHD/0257/2546) in Thailand (W.C. and C.L.).

\section{References}

1. Coussens LM and Werb Z: Inflammation and cancer. Nature 420: 860-867, 2002.

2. Sripa B, Kaewkes S, Sithithaworn P, et al: Liver fluke induces cholangiocarcinoma. PLoS Med 4: e201, 2007.

3. Sirica AE: Cholangiocarcinoma: molecular targeting strategies for chemoprevention and therapy. Hepatology 41: 5-15, 2005.

4. Vatanasapt V, Tangvoraphonkchai V, Sahaphong S, Vajrasthira S and Angsubhakorn S: A high incidence of liver cancer in Khon Kaen Province, Thailand. Southeast Asian J Trop Med Public Health 21: 489-494, 1990. 
5. Pinlaor S, Hiraku Y, Ma N, et al: Mechanism of NO-mediated oxidative and nitrative DNA damage in hamsters infected with Opisthorchis viverrini: a model of inflammation-mediated carcinogenesis. Nitric Oxide 11: 175-183, 2004.

6. Matsumoto Y, Marusawa H, Kinoshita K, et al: Helicobacter pylori infection triggers aberrant expression of activationinduced cytidine deaminase in gastric epithelium. Nat Med 13: 470-476, 2007.

7. Muramatsu M, Kinoshita K, Fagarasan S, Yamada S, Shinkai Y and Honjo T: Class switch recombination and hypermutation require activation-induced cytidine deaminase (AID), a potential RNA editing enzyme. Cell 102: 553-563, 2000.

8. Di Noia JM and Neuberger MS: Molecular mechanisms of antibody somatic hypermutation. Annu Rev Biochem 76: 1-22, 2007.

9. Kuwahara K, Fujimura S, Takahashi Y, Nakagata N, Takemori T, Aizawa S and Sakaguchi N: Germinal center-associated nuclear protein contributes to affinity maturation of B cell antigen receptor in T cell-dependent responses. Proc Natl Acad Sci USA 101: 1010-1015, 2004.

10. Sakaguchi N, Kimura T, Matsushita, et al: Generation of highaffinity antibody against $\mathrm{T}$ cell-dependent antigen in the ganp gene-transgenic mouse. J Immunol 174: 4485-4494, 2005.

11. Kuwahara K, Yoshida M, Kondo, et al: A novel nuclear phosphoprotein, GANP, is up-regulated in centrocytes of the germinal center and associated with MCM3, a protein essential for DNA replication. Blood 95: 2321-2328, 2000.

12. Abe E, Kuwahara K, Yoshida M, et al: Structure, expression, and chromosomal localization of the human gene encoding a germinal center-associated nuclear protein (GANP) that associates with MCM3 involved in the initiation of DNA replication. Gene 255: 219-227, 2000 .

13. Kuwahara K, Tomiyasu S, Fujimura S, et al: Germinal centerassociated nuclear protein (GANP) has a phosphorylationdependent DNA-primase activity that is up-regulated in germinal center regions. Proc Natl Acad Sci USA 98: 10279-10283, 2001.

14. Gallardo M, Luna, R, Erdjument-Bromage H, Tempst $\mathrm{P}$ and Aguilera A: Nab2p and the Thp1p-Sac3p complex functionally interact at the interface between transcription and mRNA metabolism. J Biol Chem 278: 24225-24232, 2003.
15. Yoshida M, Kuwahara K, Shimasaki T, Nakagata N, Matsuoka M and Sakaguchi N: GANP suppresses DNA recombination, measured by direct-repeat $\beta$-galactosidase gene construct, but does not suppress the type of recombination applying to immunoglobulin genes in mammalian cells. Genes Cells 12: 1205-1213, 2007.

16. Fujimura S, Xing Y, Takeya M, Yamashita Y, Ohshima K, Kuwahara K and Sakaguchi N: Increased expression of germinal center-associated nuclear protein RNA-primase is associated with lymphomagenesis. Cancer Res 65: 5925-5934, 2005.

17. Sripa B, Leungwattanawanit S, Nitta T, et al: Establishment and characterization of an opisthorchiasis-associated cholangiocarcinoma cell line (KKU-100). World J Gastroenterol 11: 3392-3397, 2005

18. Maruyama M, Kobayashi N, Westerman KA, et al: Establishment of a highly differentiated immortalized human cholangiocyte cell line with SV40T and hTERT. Transplantation 77: 446-451, 2004.

19. McCarthy H, Wierda WG, Barron LL, et al: High expression of activation-induced cytidine deaminase (AID) and splice variants is a distinctive feature of poor-prognosis chronic lymphocytic leukemia. Blood 101: 4903-4908, 2003.

20. Komori J, Marusawa H, Machimoto T, et al: Activation-induced cytidine deaminase links bile duct inflammation to human cholangiocarcinoma. Hepatology 47: 888-896, 2008.

21. Khan SA, Thomas HC, Toledano MB, Cox IJ and TaylorRobinson SD: p53 mutations in human cholangiocarcinoma: a review. Liver Int 25: 704-716, 2005.

22. Limpaiboon T, Krissadarak K, Sripa B, et al: Microsatellite alterations in liver fluke related cholangiocarcinoma are associated with poor prognosis. Cancer Lett 181: 215-222, 2002.

23. Bauer A and Kölling R: The SAC3 gene encodes a nuclear protein required for normal progression of mitosis. J Cell Sci 109: 1575-1583, 1996.

24. Aguilera A: Cotranscriptional mRNP assembly: from the DNA to the nuclear pore. Curr Opin Cell Biol 17: 242-250, 2005. 